Content: Key messages from research findings are delivered in brief snippets of text.

Conclusion Our videos are designed to serve two purposes: provide viewers with key evidence they can use, and link the viewer to the IWH website where they can read more information. Consultations with stakeholders on the key messages are important. One-minute research video shorts are an effective means of disseminating key research findings.

\section{TALKING ABOUT HEALTH - HOW TO COMMUNICATE ETHICAL OCCUPATIONAL HEALTH ISSUES?}

${ }^{1 S} \mathrm{~S}$ Müller, ${ }^{2} \mathrm{E}$ Kuhn, ${ }^{2} \mathrm{~A}$ Buyx, ${ }^{1} \mathrm{~L}$ Heidbrink. ${ }^{1}$ Christian-Albrechts-University Kiel, Chair of Practical Philosophy, Kiel, Germany; ${ }^{2}$ Christian-Albrechts-University Kiel, Institute of Experimental Medicine (Division of Biomedical Ethics), Kiel, Germany

\subsection{6/oemed-2018-ICOHabstracts.223}

Introduction Should we introduce fitness trackers to our company's health program? Should we, moreover, start giving positive or negative incentives for individual fitness performances (e.g. bonuses)? Since companies have felt profoundly convinced by the correlation between occupational health activities and economic success, occupational health management (OHM) becomes popular. With the rise of OHM multiple ethical problems, new to the business context, appeared. How are and how should these ethical problems be communicated inside and outside a company? One possible answer to this is corporate social responsibility (CSR). CSR already has the potential to communicate and discuss ethical corporate health issues. Only a proper framework is still missing.

Methods Qualitative interviews were completed with small, medium and large German and Swiss companies, their stakeholders, customers and industry representatives. They all were asked questions regarding ethical communication strategies inside and outside companies and in particular questions about structural intersections between OHM and CSR.

Result The study shows that, if it comes to occupational health, most companies do not use the existing CSR-tools to communicate ethical heath issues. Usually the few existing intersections between OHM and CSR are not meant to point out or discuss ethical problems within OHM, but to promote the company's image. Not surprisingly, the stakeholders as well as the industry representatives emphasise the companies' voluntary assumption of responsibility.

Discussion Most CSR strategies already contain ways to address and communicate ethical problems (e.g. whistle-blower hotline, companies' suggestion systems). Whatsoever, these strategies usually are not applied to OHM. The social and ethical arguments considered in CSR as well as its strong attachment to the company's strategy could pave the way for an ethical OHM. In reverse, an ethical OHM will arguably have a positive reinforcement on CSR activities.
764

HEAT STRESS MANGMENT PROGRAM OF SOHAR ALUMINIUM - TRANSLATING SCIENTIFIC CONCEPTS AND TECHNOLOY INTO EFFECTIVE WORK PLACE INTERVENTION AND MANAGEMENT

${ }^{1}$ Gummanur T Manjunath, ${ }^{2}$ Rajeev Aravindhakshan, ${ }^{1}$ Shyju Varghese. 'Sohar Aluminium Health Centre; ${ }^{2}$ Pushpagiri Medical College and Research Centre

\subsection{6/oemed-2018-ICOHabstracts.224}

Introduction Environmental conditions in Sultanate of Oman and other Arabian Gulf countries are some of the harshest in the world. Primary Aluminium smelting requires enormous amounts of energy, in the form of affordable and uninterrupted supply of electricity. Workers engaged in physically demanding and manual tasks in hot environments are vulnerable to heat illness, besides being at risk being easily fatigued leading to work place injuries.

Combination of work place and physiological monitoring procedures incorporated into a structured Heat Stress Monitoring Program implemented by the Medical Team headed by the main author supported by and in collaboration with external consultant(co-author) have lead to discernible and sustainable occupational health improvements in the work place

Strategy While work place monitoring requires measuring relevant thermal index that is easy to measure and interpret on the spot, physiological monitoring involves measurement of parameters like urine specific gravity, heart rate and body temperature etc by a licensed healthcare professional at work place. Major challenge however is how to effectively communicate the work place risks, results of measurements mentioned earlier and preventative and remedial measures to the work force, in order to translate the concepts into effective occupational health intervention. Communicating with workers needs to be void of technical jargon and keeping that in mind, Sohar Aluminium Medial Team introduced a revised Heat Stress Management Program incorporating work site Hydration Monitoring procedures and communication strategy. The idea or concept of 'issuing cards on the spot' used in football game to educate and discipline the players was incorporated into Heat Stress Management program, along with Traffic signalling colour codes. Hydration tested workers were classified into three classes as 'Normally Hydrated (with USG <1.019), 'Under Hydrated (with USG 1.020-1.029) and 'Dehydrated' with urine specific gravity results tested with a refractometer. Normally hydrated workers were given a green card, Under hydrated workers a yellow card and Dehydrated workers a Red Card, with relevant messages explaining the result of testing and measures needed to be taken by the workers.

This proactive approach in the program lead to effectively prevent and mitigate heat stress at Sohar Aluminium and made a major and significant difference to the workers and contractors and helped in reducing the incidence of heat illnesses. 

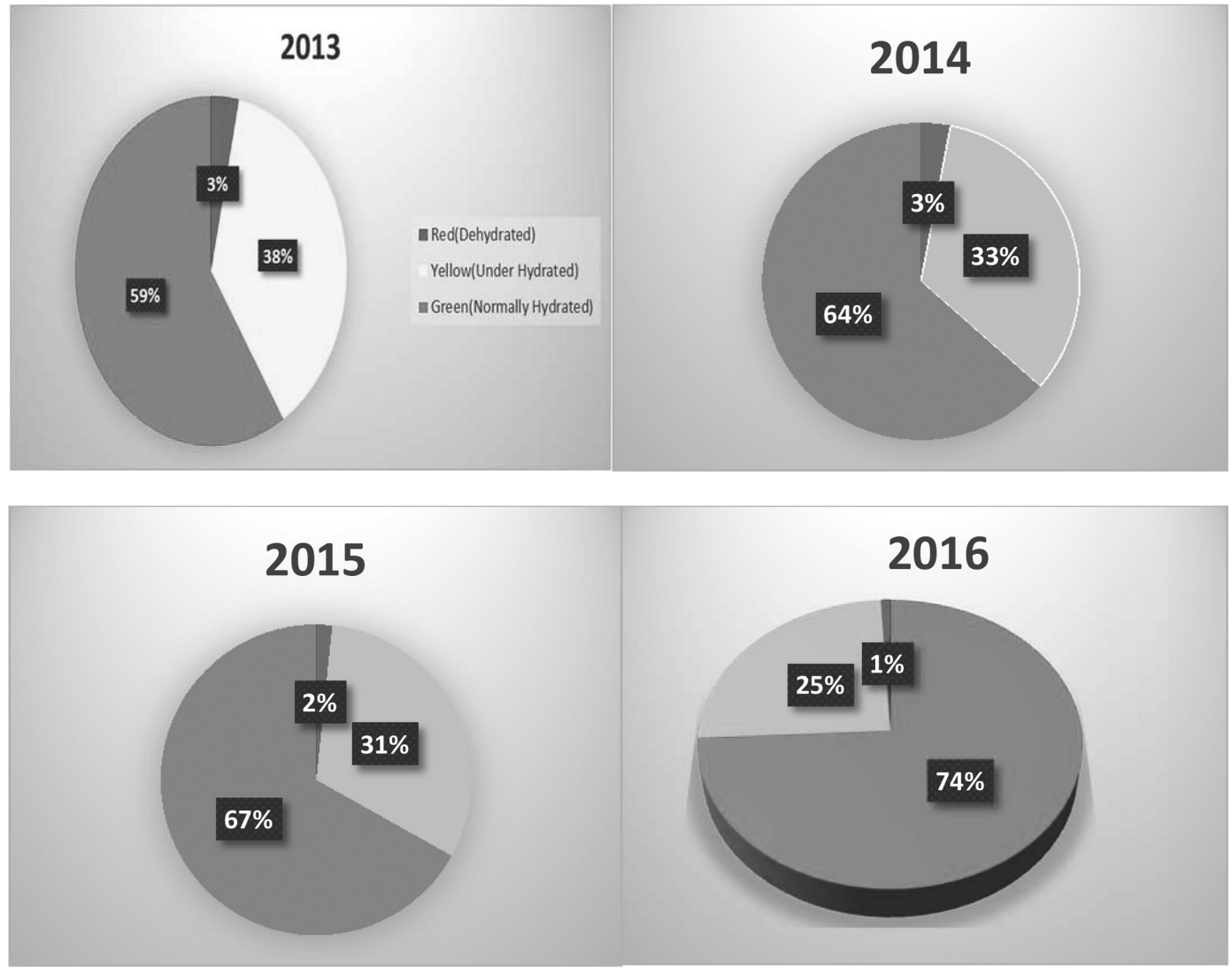

Abstract 764 Figure 1 Hydration status in tested population 2013, 2014, 2105 \& 2016 (in\%)

Results of urine specific gravity testing were analysed daily, weekly and monthly and shared with relevant leaders in management as well. Work place measurements of thermal index parameters like temperature, humidity etc. were analysed which helped in creation of 'Heat Stress Risk Map' for specific work areas and the entire organisation. These measures helped area managers to plan work activities prudently during summer months. Statistical Analyses results on the number of green cards, yellow cards and red cards were incorporated into management lean Board discussions.

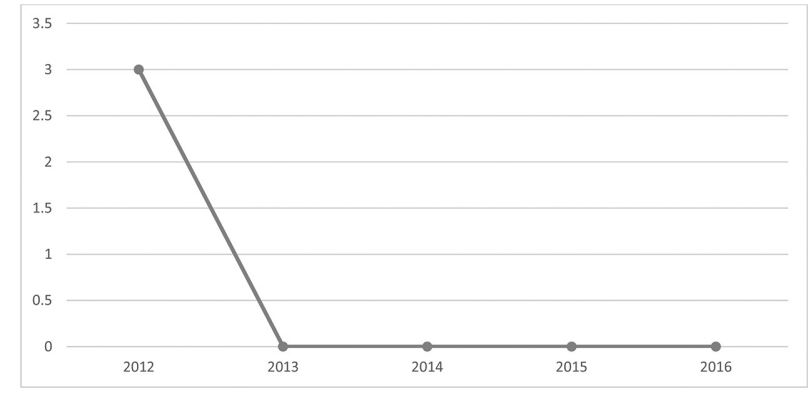

Abstract 764 Figure 2 Recordable heat illness cases (2013-2016)

Conclusion These measures among other supportive measures resulted in significant reduction of cases of heat illness, particularly elimination of recordable heat illness cases from 2013 through 2016 (figures 1 and 2).

\section{COMMUNICATING WORKPLACE HEALTH \& WELLBEING IN THE HEALTH SERVICE EXECUTIVE (HSE)}

${ }^{1}$ Emer Carroll, ${ }^{2}$ Nodlaig Carroll, ${ }^{1}$ Noelle Dalton, ${ }^{1}$ Fiona Dardis, ${ }^{3}$ Sally Downing. ${ }^{1}$ National Health and Safety Function (NHSF), Health Service Executive (HSE); ${ }^{2}$ Organizational Psychology Unit, Health Service Executive (HSE); ${ }^{3}$ National Communications Division, Health Service Executive (HSE)

\subsection{6/oemed-2018-ICOHabstracts.225}

Introduction The Health Service Executive (HSE) recognise health and wellbeing as a continuum of care for staff throughout their working life. The Workplace Health and Wellbeing (WHW) Division, established in May 2016, aims to raise awareness on the importance of safeguarding health and wellbeing at work by providing supports, tools and information that encourage a healthy working life.

To achieve this goal, a multidisciplinary project team developed an effective communication framework highlighting the support services and initiatives available through the services of Health and Safety, Occupational Health, Employee Assistance and Counselling Services and Health Promotion and Improvement, under the WHW Division.

Methods The project team reviewed international and national communication practices for health and wellbeing within the workplace. Throughout the project, the team engaged with key stakeholders and employees to identify what information staff required and how this information should be collated and governed. Using the HSE Change Management framework, agreement was reached on website design, content and promotion. 